International Journal of Pure and Applied Mathematics

Volume 109 No. $3 \quad 2016,619-629$

ISSN: 1311-8080 (printed version); ISSN: 1314-3395 (on-line version)

url: http://www.ijpam.eu

doi: 10.12732/ijpam.v109i3.11

\title{
FIXED POINTS FOR COMPATIBLE MAPPINGS IN MULTIPLICATIVE METRIC SPACES
}

\author{
Parveen Kumar ${ }^{1}$, Sanjay Kumar ${ }^{2}$, Shin Min Kang ${ }^{3}$, \\ Chahn Yong Jung ${ }^{4}$ \\ ${ }^{1,2}$ Departement of Mathematics \\ Deenbandhu Chhotu Ram University of Science and Technology \\ Murthal, Sonepat 131039, Haryana, INDIA \\ ${ }^{3}$ Department of Mathematics and RINS \\ Gyeongsang National University \\ Jinju, 52828, KOREA \\ ${ }^{4}$ Department of Business Administration \\ Gyeongsang National University \\ Jinju 52828, KOREA
}

\begin{abstract}
In this paper, we proved the common fixed point result for compatible mappings in multiplicative metric spaces.
\end{abstract}

AMS Subject Classification: 47H10, 54H 25

Key Words: multiplicative metric spaces, compatible mappings

\section{Introduction and Preliminaries}

It is well know that the set of positive real numbers $\mathbb{R}_{+}$is not complete according to the usual metric. To overcome this problem, in 2008, Bashirov et al. [1] introduced the concept of multiplicative metric spaces as follows:

Received: $\quad$ July 7,2016

Revised: $\quad$ August 18, 2016

Published: September 30, 2016

${ }^{\S}$ Correspondence author (c) 2016 Academic Publications, Ltd. url: www.acadpubl.eu 
Definition 1.1. Let $X$ be a nonempty set. A multiplicative metric is a mapping $d: X \times X \rightarrow \mathbb{R}_{+}$satisfying the following conditions:

(i) $d(x, y) \geq 1$ for all $x, y \in X$ and $d(x, y)=1$ if and only if $x=y$;

(ii) $d(x, y)=d(y, x)$ for all $x, y \in X$;

(iii) $d(x, y) \leq d(x, z) \cdot d(z, y)$ for all $x, y, z \in X$ (multiplicative triangle inequality).

Then the mapping $d$ together with $X$, that is, $(X, d)$ is a multiplicative metric space.

Example 1.2. ([3]) Let $\mathbb{R}_{+}^{n}$ be the collection of all $n$-tuples of positive real numbers. Let $d^{*}: \mathbb{R}_{+}^{n} \times \mathbb{R}_{+}^{n} \rightarrow \mathbb{R}$ be defined as follows:

$$
d^{*}(x, y)=\left|\frac{x_{1}}{y_{1}}\right|^{*} \cdot\left|\frac{x_{2}}{y_{2}}\right|^{*} \cdots\left|\frac{x_{n}}{y_{n}}\right|^{*},
$$

where $x=\left(x_{1}, \ldots, x_{n}\right), y=\left(y_{1}, \ldots, y_{n}\right) \in \mathbb{R}_{+}^{n}$ and $|\cdot|^{*}: \mathbb{R}_{+} \rightarrow \mathbb{R}_{+}$is defined by

$$
|a|^{*}= \begin{cases}a & \text { if } a \geq 1, \\ \frac{1}{a} & \text { if } a<1 .\end{cases}
$$

Then it is obvious that all conditions of a multiplicative metric are satisfied. Therefore $\left(\mathbb{R}_{+}^{n}, d^{*}\right)$ is a multiplicative metric space.

One can refer to [3] for detailed a multiplicative metric topology.

Definition 1.3. Let $(X, d)$ be a multiplicative metric space. Then a sequence $\left\{x_{n}\right\}$ in $X$ said to be

(1) a multiplicative convergent to $x$ if for every multiplicative open ball $B_{\epsilon}(x)=\{y \mid d(x, y)<\epsilon\}, \epsilon>1$, there exists $N \in \mathbb{N}$ such that $x_{n} \in B_{\epsilon}(x)$ for all $n \geq N$, that is, $d\left(x_{n}, x\right) \rightarrow 1$ as $n \rightarrow \infty$.

(2) a multiplicative Cauchy sequence if for all $\epsilon>1$, there exists $N \in \mathbb{N}$ such that $d\left(x_{n}, x_{m}\right)<\epsilon$ for all $m, n \geq N$, that is, $d\left(x_{n}, x_{m}\right) \rightarrow 1$ as $n, m \rightarrow \infty$.

(3) We call a multiplicative metric space complete if every multiplicative Cauchy sequence in it is multiplicative convergent to $x \in X$.

In 2012, Özavsar and Çevikel [3] gave the concept of multiplicative contractive mappings and proved some fixed point theorem of such mappings in a multiplicative metric space.

Definition 1.4. Let $f$ be a mapping of a multiplicative metric space $(X, d)$ into itself. Then $f$ is said to be a multiplicative contraction if there exists a real number $\lambda \in[0,1)$ such that

$$
d(f x, f y) \leq d^{\lambda}(x, y) \quad \text { for all } x, y \in X .
$$


In 2015, Kang et al. [2] introduced the notion of compatible mappings as follows:

Definition 1.5. Let $f$ and $g$ be mappings of a multiplicative metric space $(X, d)$ Then $f$ and $g$ are called compatible if

$$
\lim _{n \rightarrow \infty} d\left(f g x_{n}, g f x_{n}\right)=1
$$

whenever $\left\{x_{n}\right\}$ is a sequence in $X$ such that $\lim _{n \rightarrow \infty} f x_{n}=\lim _{n \rightarrow \infty} g x_{n}=t$ for some $t \in X$,

Now we give some properties related to compatible mappings and its variants in a multiplicative metric space, see [2].

Proposition 1.6. Let $f$ and $g$ be compatible mappings of a multiplicative metric space $(X, d)$ into itself. If $f t=g t$ for some $t \in X$, then $f g t=f f t=$ $g g t=g f t$.

Proposition 1.7. Let $f$ and $g$ be compatible mappings of a multiplicative metric space $(X, d)$ itself. Suppose that $\lim _{n \rightarrow \infty} f x_{n}=\lim _{n \rightarrow \infty} g x_{n}=t$ for some $t \in X$. Then

(i) $\lim _{n \rightarrow \infty} g f x_{n}=f t$ if $f$ is continuous at $t$.

(ii) $\lim _{n \rightarrow \infty} f g x_{n}=g t$ if $g$ is continuous at $t$.

(iii) $f g t=g f t$ and $f t=g t$ if $f$ and $g$ are continuous at $t$.

\section{Main Results}

Now we give the following theorem for compatible mappings.

Theorem 2.1. Let $A, B, S$ and $T$ be mappings of a complete multiplicative metric space $(X, d)$ satisfying the following conditions

$\left(C_{1}\right) \quad S X \subset B X, \quad T X \subset A X$

$$
d(S x, T y) \leq M^{\lambda}(x, y)
$$


for each $x, y \in X$ and $\lambda \in(0,1 / 2)$, where

$$
\begin{aligned}
& M(x, y) \\
& =\max \left\{d(A x, S x), d(B y, T y), d(B y, A x),(d(A x, T y) \cdot d(B y, S x))^{1 / 2},\right. \\
& \quad \min \left\{\frac{d(A x, S x) \cdot d(B y, T y)}{d(A x, B y)}, \frac{d(A x, T y) \cdot d(B y, S x)}{d(A x, B y)},\right. \\
& \left.\left.\quad \frac{d(A x, T y) \cdot d(B y, S x)}{d(S x, T y)}\right\}\right\}
\end{aligned}
$$

$\left(C_{3}\right)$ one of $A, B, S$ and $T$ is continuous.

Assume that the pairs $A, S$ and $B, T$ are compatible. Then $A, B, S$ and $T$ have a unique common fixed point.

Proof. Let $x_{0} \in X$ be an arbitrary point. Since $S X \subset B X$ and $T X \subset A X$, there exists $x_{1} \in X$ such that $S x_{0}=B x_{1}=y_{0}$ and for this point $x_{1}$, there exists $x_{2} \in X$ such that $T x_{1}=A x_{2}=y_{1}$. Continuing in this way, we can construct a sequence $\left\{y_{n}\right\}$ such that

$$
y_{2 n+1}=T x_{2 n+1}=A x_{2 n+2} ; \quad y_{2 n}=S x_{2 n}=B x_{2 n+1} .
$$

From $\left(C_{2}\right)$ by putting $x=x_{2 n}$ and $y=x_{2 n+1}$, we have

$$
d\left(y_{2 n}, y_{2 n+1}\right)=d\left(S x_{2 n}, T x_{2 n+1}\right) \leq M^{\lambda}\left(x_{2 n}, x_{2 n+1}\right)
$$

where

$$
\begin{aligned}
M & \left(x_{2 n}, x_{2 n+1}\right)=\max \left\{d\left(A x_{2 n}, S x_{2 n}\right), d\left(B x_{2 n+1}, T x_{2 n+1}\right), d\left(B x_{2 n+1}, A x_{2 n}\right),\right. \\
& \left(d\left(A x_{2 n}, T x_{2 n+1}\right) \cdot d\left(B x_{2 n+1}, S x_{2 n}\right)\right)^{1 / 2}, \\
& \min \left\{\frac{d\left(A x_{2 n}, S x_{2 n}\right) \cdot d\left(B x_{2 n+1}, T x_{2 n+1}\right)}{d\left(A x_{2 n}, B x_{2 n+1}\right)},\right. \\
& \frac{d\left(A x_{2 n}, T x_{2 n+1}\right) \cdot d\left(B x_{2 n+1}, S x_{2 n}\right)}{d\left(A x_{2 n}, B x_{2 n+1}\right)}, \\
& \left.\left.\frac{d\left(A x_{2 n}, T x_{2 n+1}\right) \cdot d\left(B x_{2 n+1}, S x_{2 n}\right)}{d\left(S x_{2 n}, T x_{2 n+1}\right)}\right\}\right\} \\
= & \max \left\{d\left(y_{2 n}, y_{2 n-1}\right), d\left(y_{2 n+1}, y_{2 n}\right), d\left(y_{2 n}, y_{2 n-1}\right),\right. \\
& \left(d\left(y_{2 n}, y_{2 n}\right) \cdot d\left(y_{2 n+1}, y_{2 n-1}\right)\right)^{1 / 2}, \min \left\{\frac{d\left(y_{2 n}, y_{2 n-1}\right) \cdot d\left(y_{2 n}, y_{2 n+1}\right)}{d\left(y_{2 n}, y_{2 n-1}\right)}\right.
\end{aligned}
$$




$$
\begin{aligned}
& \left.\left.\frac{d\left(y_{2 n}, y_{2 n}\right) \cdot d\left(y_{2 n+1}, y_{2 n-1}\right)}{d\left(y_{2 n}, y_{2 n-1}\right)}, \frac{d\left(y_{2 n} y_{2 n}\right) \cdot d\left(y_{2 n+1}, y_{2 n-1}\right)}{d\left(y_{2 n}, y_{2 n+1}\right)}\right\}\right\} \\
= & \max \left\{d\left(y_{2 n}, y_{2 n-1}\right), d\left(y_{2 n+1}, y_{2 n}\right)\right\} .
\end{aligned}
$$

Now if $d\left(y_{2 n+1}, y_{2 n}\right)>d\left(y_{2 n-1}, y_{2 n}\right)$, then $M\left(x_{2 n}, x_{2 n+1}\right)=d\left(y_{2 n+1}, y_{2 n}\right)$. Then we have,

$$
d\left(y_{2 n}, y_{2 n+1}\right) \leq d^{\lambda}\left(y_{2 n}, y_{2 n+1}\right)
$$

which is a contradiction as $\lambda \in(0,1 / 2)$. So, $d\left(y_{2 n}, y_{2 n-1}\right) \geq d\left(y_{2 n+1}, y_{2 n}\right)$, which implies that $M\left(x_{2 n}, x_{2 n+1}\right)=d\left(y_{2 n-1}, y_{2 n}\right)$ and hence

$$
d\left(y_{2 n}, y_{2 n+1}\right) \leq d^{\lambda}\left(y_{2 n-1}, y_{2 n}\right)
$$

Similarly, we have

$$
d\left(y_{2 n-1}, y_{2 n}\right) \leq d^{\lambda}\left(y_{2 n-2}, y_{2 n-1}\right)
$$

Hence, in general we get

$$
\begin{aligned}
d\left(y_{n}, y_{n+1}\right) & \leq d^{\lambda}\left(y_{n-1}, y_{n}\right) \\
& \leq d^{\lambda^{2}}\left(y_{n-2}, y_{n-1}\right) \\
& \leq \cdots \leq d^{\lambda^{n}}\left(y_{0}, y_{1}\right)
\end{aligned}
$$

Let $m, n \in \mathbb{N}$ with $m>n$. Then

$$
\begin{aligned}
d\left(y_{m}, y_{n}\right) & \leq d\left(y_{m}, y_{m-1}\right) \cdot d\left(y_{m-1}, y_{m-2}\right) \cdots d\left(y_{n+1}, y_{n}\right) \\
& \left.\leq d^{\lambda^{m-1}}\left(y_{0}, y_{1}\right)\right) \cdot d^{\lambda^{m-2}}\left(y_{0}, y_{1}\right) \cdots d^{\lambda^{n}}\left(y_{0}, y_{1}\right) \\
& \leq d^{\frac{\lambda^{n}}{1-\lambda}}\left(y_{0}, y_{1}\right) .
\end{aligned}
$$

Taking $n \rightarrow \infty$, we have $d\left(y_{m}, y_{n}\right) \leq 1$ and hence $\lim _{n \rightarrow \infty} d\left(y_{m}, y_{n}\right)=1$. Thus $\left\{y_{n}\right\}$ is a multiplicative Cauchy sequence in $X$ and hence it converges to some point $z \in X$. Consequently, the subsequence $\left\{A x_{2 n}\right\},\left\{B x_{2 n+1}\right\}$, $\left\{T x_{2 n+1}\right\}$ and $\left\{S x_{2 n}\right\}$ of the sequence $\left\{y_{n}\right\}$ also converges to $z$.

Now suppose that $A$ is continuous. Since $A$ and $S$ are compatible on $X$, it follows from Proposition 1.7 that

$$
A A x_{2 n} \rightarrow A z, \quad S A x_{2 n} \rightarrow A z \quad \text { as } n \rightarrow \infty .
$$

Now putting $x=A x_{2 n}$ and $y=x_{2 n+1}$ in $\left(C_{2}\right)$, we have

$$
d\left(S A x_{2 n}, T x_{2 n+1}\right) \leq M^{\lambda}\left(A x_{2 n}, x_{2 n+1}\right),
$$


where

$$
\begin{aligned}
& M\left(A x_{2 n}, x_{2 n+1}\right) \\
& =\max \left\{d\left(A A x_{2 n}, S A x_{2 n}\right), d\left(B x_{2 n+1}, T x_{2 n+1}\right), d\left(B x_{2 n+1}, A A x_{2 n}\right),\right. \\
& \left(d\left(A A x_{2 n}, T x_{2 n+1}\right) \cdot d\left(B x_{2 n+1}, S A x_{2 n}\right)\right)^{1 / 2}, \\
& \quad \min \left\{\frac{d\left(A A x_{2 n}, S A x_{2 n}\right) \cdot d\left(B x_{2 n+1}, T x_{2 n+1}\right)}{d\left(A A x_{2 n}, B x_{2 n+1}\right)},\right. \\
& \quad \frac{d\left(A A x_{2 n}, T x_{2 n+1}\right) \cdot d\left(B x_{2 n+1}, S A x_{2 n}\right)}{d\left(A A x_{2 n}, B x_{2 n+1}\right)}, \\
& \left.\left.\quad \frac{d\left(A A x_{2 n}, T x_{2 n+1}\right) \cdot d\left(B x_{2 n+1}, S A x_{2 n}\right)}{d\left(S A x_{2 n}, T x_{2 n+1}\right)}\right\}\right\} .
\end{aligned}
$$

Letting $n \rightarrow \infty$, we have

$$
\begin{aligned}
& \lim _{n \rightarrow \infty} M\left(A x_{2 n}, x_{2 n+1}\right) \\
& =\max \left\{d(A z, A z), d(z, z), d(z, A z),(d(A z, z) \cdot d(z, A z))^{1 / 2},\right. \\
& \left.\quad \min \left\{\frac{d(A z, A z) \cdot d(z, z)}{d(A z, z)}, d(z, A z), d(A z, z)\right\}\right\} \\
& =d(z, A z) .
\end{aligned}
$$

Hence

$$
d(z, A z) \leq d^{\lambda}(z, A z)
$$

which a contradiction, we get $z=A z$.

Next putting $x=z$ and $y=x_{2 n+1}$ in $\left(C_{2}\right)$, we have

$$
d\left(S z, T x_{2 n+1}\right) \leq M^{\lambda}\left(z, x_{2 n+1}\right),
$$

where

$$
\begin{aligned}
& M\left(z, x_{2 n+1}\right) \\
& =\max \left\{d(A z, S z), d\left(B x_{2 n+1}, T x_{2 n+1}\right), d\left(B x_{2 n+1}, A z\right),\right. \\
& \quad\left(d\left(A z, T x_{2 n+1}\right) \cdot d\left(B x_{2 n+1}, S z\right)\right)^{1 / 2}, \\
& \quad \min \left\{\frac{d(A z, S z) \cdot d\left(B x_{2 n+1}, T x_{2 n+1}\right)}{d\left(A z, B x_{2 n+1}\right)},\right. \\
& \left.\left.\quad \frac{d\left(A z, T x_{2 n+1}\right) \cdot d\left(B x_{2 n+1}, S z\right)}{d\left(A z, B x_{2 n+1}\right)}, \frac{d\left(A z, T x_{2 n+1}\right) \cdot d\left(B x_{2 n+1}, S z\right)}{d\left(S z, T x_{2 n+1}\right)}\right\}\right\} .
\end{aligned}
$$


Letting $n \rightarrow \infty$, we have

$$
\begin{aligned}
& \lim _{n \rightarrow \infty} M\left(z, x_{2 n+1}\right) \\
& =\max \left\{d(z, S z), d(z, z), d(z, z),(d(z, z) \cdot d(z, S z))^{1 / 2}\right. \\
& \quad \min \{d(z, S z), d(z, S z), d(z, z)\}\} \\
& =d(S z, z) .
\end{aligned}
$$

Then we have

$$
d(S z, z) \leq d^{\lambda}(S z, z)
$$

which a contradiction, we get $S z=z$. Since $S X \subset B X$, there exists a point $u \in X$ such that $z=S z=B u$.

Again putting $x=z$ and $y=u$ in $\left(C_{2}\right)$, we have

$$
d(z, T u)=d(S z, T u) \leq M^{\lambda}(z, u)
$$

where

$$
\begin{aligned}
M(z, u)= & \max \{d(A z, S z), d(B u, T u), d(B u, A z) \\
& (d(A z, T u) \cdot d(B u, S z))^{1 / 2}, \min \left\{\frac{d(A z, S z) \cdot d(B u, T u)}{d(A z, B u)},\right. \\
& \left.\left.\quad \frac{d(A z, T u), d(B u, S z)}{d(A z, B u)}, \frac{d(A z, T u) \cdot d(B u, S z)}{d(S z, T u)}\right\}\right\} \\
= & \max \left\{1, d(z, T u), 1, d^{1 / 2}(z, T u), 1\right\} \\
= & d(z, T u) .
\end{aligned}
$$

Then

$$
d(z, T u) \leq d^{\lambda}(z, T u)
$$

which a contradiction. This implies that $z=T u$. Since $B$ and $T$ are compatible on $X$ and $B u=T u=z$, by Proposition 1.6, BTu=TBu and hence $B z=$ $B T u=T B u=T z$.

Also, we have

$$
d(z, B z)=d(S z, T z) \leq M^{\lambda}(z, z)
$$


where

$$
\begin{aligned}
& M(z, z) \\
& =\max \{d(A z, S z), d(B z, T z), d(B z, A z), \\
& \quad(d(A z, T z) \cdot d(B z, S z))^{1 / 2}, \min \left\{\frac{d(A z, S z) \cdot d(B z, T z)}{d(A z, B z)},\right. \\
& \left.\left.\quad \frac{d(A z, T z), d(B z, S z)}{d(A z, B z)}, \frac{d(A z, T z) \cdot d(B z, S z)}{d(S z, T z)}\right\}\right\} \\
& =\max \left\{1,1, d(B z, z), d(B z, z), \min \left\{\frac{1}{d(z, B z)}, d(B z, z), d(B z, z)\right\}\right\} \\
& =d(B z, z) .
\end{aligned}
$$

This implies that $z=B z$. Hence, $z=B z=T z=A z=S z$. Therefore, $z$ is a common fixed point of $A, B, S$ and $T$.

Similarly, we can also complete the proof when $B$ is continuous.

Next suppose that $S$ is continuous. Since $A$ and $S$ are compatible on $X$, it follows that

$$
S^{2} x_{2 n} \rightarrow S z, \quad A S x_{2 n} \rightarrow S z \quad \text { as } n \rightarrow \infty .
$$

Now putting $x=S x_{2 n}$ and $y=x_{2 n+1}$ in $\left(C_{2}\right)$

$$
d\left(S S x_{2 n}, T x_{2 n+1}\right) \leq M^{\lambda}\left(S x_{2 n}, x_{2 n+1}\right),
$$

where

$$
\begin{aligned}
& M\left(S x_{2 n}, x_{2 n+1}\right) \\
& =\max \left\{d\left(A S x_{2 n}, S S x_{2 n}\right), d\left(B x_{2 n+1}, T x_{2 n+1}\right), d\left(B x_{2 n+1}, A S x_{2 n}\right),\right. \\
& \left(d\left(A S x_{2 n}, T x_{2 n+1}\right) \cdot d\left(B x_{2 n+1}, S S x_{2 n}\right)\right)^{1 / 2}, \\
& \min \left\{\frac{d\left(A S x_{2 n}, S S x_{2 n}\right) \cdot d\left(B x_{2 n+1}, T x_{2 n+1}\right)}{d\left(A S x_{2 n}, B x_{2 n+1}\right)},\right. \\
& \quad \frac{d\left(A S x_{2 n}, T x_{2 n+1}\right) \cdot d\left(B x_{2 n+1}, S S x_{2 n}\right)}{d\left(A S x_{2 n}, B x_{2 n+1}\right)}, \\
& \left.\left.\quad \frac{d\left(A S x_{2 n}, T x_{2 n+1}\right) \cdot d\left(B x_{2 n+1}, S S x_{2 n}\right)}{d\left(S S x_{2 n}, T x_{2 n+1}\right)}\right\}\right\} .
\end{aligned}
$$


Letting $n \rightarrow \infty$, we get

$$
\begin{aligned}
\lim _{n \rightarrow \infty} M\left(S x_{2 n}, x_{2 n+1}\right)= & \max \{d(S z, S z), d(z, z), d(z, S z), d(z, S z), \\
& \left.\min \left\{\frac{1}{d(z, S z)}, d(z, S z), d(z, S z)\right\}\right\} \\
= & d(z, S z) .
\end{aligned}
$$

This implies that $S z=z$. Since $S X \subset B X$, there exists a point $v \in X$ such that $z=S z=B v$.

Now putting $x=S x_{2 n}$ and $y=v$ in $\left(C_{2}\right)$

$$
d\left(S S x_{2 n}, T v\right) \leq M^{\lambda}\left(S x_{2 n}, v\right)
$$

where

$$
\begin{aligned}
& M\left(S x_{2 n}, v\right) \\
& =\max \left\{d\left(A S x_{2 n}, S S x_{2 n}\right), d(B v, T v), d\left(B v, A S x_{2 n}\right)\right. \\
& \quad\left(d\left(A S x_{2 n}, T v\right) \cdot d\left(B v, S S x_{2 n}\right)\right)^{1 / 2}, \min \left\{\frac{d\left(A S x_{2 n}, S S x_{2 n}\right) \cdot d(B v, T v)}{d\left(A S x_{2 n}, B v\right)},\right. \\
& \left.\left.\quad \frac{d\left(A S x_{2 n}, T v\right) \cdot d\left(B v, S S x_{2 n}\right)}{d\left(A S x_{2 n}, B v\right)}, \frac{d\left(A S x_{2 n}, T v\right) \cdot d\left(B v, S S x_{2 n}\right)}{d\left(S S x_{2 n}, T v\right)}\right\}\right\} .
\end{aligned}
$$

Letting $n \rightarrow \infty$, we get

$$
\begin{aligned}
& \lim _{n \rightarrow \infty} M\left(S x_{2 n}, v\right) \\
& =\max \left\{1, d(z, T v), 1, d^{1 / 2}(z, T v), \min \{d(z, T v), d(z, T v), 1\}\right\} \\
& =d(z, T v)
\end{aligned}
$$

This implies that $u=T v$. Since $B$ and $T$ are compatible on $X$ and $B v=T v=z$, $B T v=T B v$ and hence $B z=B T v=T B v=T z$.

Now putting $x=x_{2 n}$ and $y=z$ in $\left(C_{2}\right)$

$$
d\left(S x_{2 n}, T z\right) \leq M^{\lambda}\left(x_{2 n}, z\right)
$$


where

$$
\begin{aligned}
& M\left(x_{2 n}, z\right) \\
& =\max \left\{d\left(A x_{2 n}, S x_{2 n}\right), d(B z, T z), d\left(B u, A x_{2 n}\right),\right. \\
& \quad\left(d\left(A x_{2 n}, T z\right) \cdot d\left(B z, S x_{2 n}\right)\right)^{1 / 2}, \min \left\{\frac{d\left(A x_{2 n}, S x_{2 n}\right) \cdot d(B z, T z)}{d\left(A x_{2 n}, B z\right)},\right. \\
& \left.\left.\quad \frac{d\left(A x_{2 n}, T z\right) \cdot d\left(B z, S x_{2 n}\right)}{d\left(A x_{2 n}, B z\right)}, \frac{d\left(A x_{2 n}, T z\right) \cdot d\left(B z, S x_{2 n}\right)}{d\left(S x_{2 n}, T z\right)}\right\}\right\} .
\end{aligned}
$$

Letting $n \rightarrow \infty$, we get

$$
\begin{aligned}
& \lim _{n \rightarrow \infty} M\left(x_{2 n}, z\right) \\
& =\max \left\{1,1, d(T z, z), d(T z, z), \min \left\{\frac{1}{d(z, T z)}, d(T z, z), d(T z, z)\right\}\right\} \\
& =d(T z, z) .
\end{aligned}
$$

This implies that $T z=z$. Since $T X \subset A X$, there exists a point $w \in X$ such that $z=T z=A w$.

Now putting $x=w$ and $y=z$ in $\left(C_{2}\right)$

$$
d(S w, T z) \leq M^{\lambda}(w, z)
$$

where

$$
\begin{aligned}
& M(w, z) \\
& =\max \{d(A w, S w), d(B z, T z), d(B z, A w), \\
& \quad(d(A w, T z) \cdot d(B z, S w))^{1 / 2}, \min \left\{\frac{d(A w, S w) \cdot d(B z, T z)}{d(A w, B z)},\right. \\
& \left.\left.\quad \frac{d(A w, T z) \cdot d(B z, S w)}{d(A w, B z)}, \frac{d(A w, T z) \cdot d(B z, S w)}{d(S w, T z)}\right\}\right\} \\
& =\max \left\{d(z, S w), 1,1, d^{1 / 2}(z, S w), \min \{d(z, S w), d(z, S w), 1\}\right\} \\
& =d(z, S w) .
\end{aligned}
$$

This implies that $S w=z$. Since $A$ and $S$ are compatible on $X$ and $S w=A w=$ $z, A S w=S A w$ and hence $A z=A S w=S A w=S z$. That is, $z=A z=S z=$ $B z=T z$. Therefore, $z$ is a common fixed point of $A, B, S$ and $T$.

Similarly, we can complete the proof when $T$ is continuous. 
Finally, suppose that $z$ and $w$ are two common fixed points of $A, B, S$ and $T$.

Now putting $x=z$ and $y=w$ in $\left(C_{2}\right)$

$$
d(z, w)=d(S z, T w) \leq M^{\lambda}(z, w)
$$

where

$$
\begin{aligned}
& M(z, w) \\
& =\max \{d(A z, S z), d(B w, T w), d(B w, A z) \\
& \quad(d(A z, T w) \cdot d(B w, S z))^{1 / 2}, \min \left\{\frac{d(A z, S z) \cdot d(B w, T w)}{d(A z, B w)}\right. \\
& \left.\left.\quad \frac{d(A z, T w) \cdot d(B w, S z)}{d(A z, B w)}, \frac{d(A z, T w) \cdot d(B w, S z)}{d(S z, T w)}\right\}\right\} \\
& =\max \left\{1,1, d(z, w), d(z, w), \min \left\{\frac{1}{d(z, w)}, d(z, w), d(z, w)\right\}\right\} \\
& =d(z, w),
\end{aligned}
$$

which implies that $z=w$. Therefore, $A, B, S$ and $T$ have a unique common fixed point in $X$. This completes the proof.

\section{References}

[1] A.E. Bashirov, E.M. Kurplnara, A. Ozyapici, Multiplicative calculus and its applicatiopns, J. Math. Anal. Appl., 337 (2008), 36-48, doi: 10.1016/j.jmaa.2007.03.081.

[2] S.M. Kang, P. Kumar, S. Kumar, P. Nagpal, S.K. Garg, Common fixed points for compatible mappings and its variants in multiplicative metric spaces, Int. J. Pure Appl. Math., 102 (2015), 383-406. doi: 10.12732/ijpam.v102i2.14.

[3] M. Özavsar, A.C. Çevikel, Fixed points of multiplicative contraction mappings on multiplicative metric spaces, ArXiv: 1205.5131v1 [math.GM] (2012), 14 pages. 
